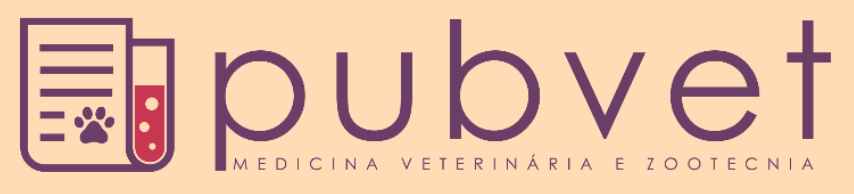

https://doi.org/10.31533/pubvet.v14n3a535.1-7

\title{
Enteroparasitoses e fatores de risco em escolares de uma cidade do Nordeste do Brasil
}

\author{
Israel Gomes de Amorim Santos ${ }^{10}$, Janaí da Conceição Silva ${ }^{10}$, Rafael Alves $\operatorname{Ramos}^{10}$, \\ Romenito Guilherme de Melo $^{1}{ }^{\ominus}$, Sheilla da Conceição Gomes $^{1 \bullet}$, Mayke Bezerra Alencar ${ }^{30}$, \\ João Paulo Vieira Machado ${ }^{1}{ }^{\ominus}$, Glória Isabel Lisboa da Silva ${ }^{1} \bullet$, Ivisson abreu Damasceno ${ }^{10}$, \\ Dharliton Soares Gomes ${ }^{4}$, Rosália Elen Santos $\operatorname{Ramos}^{4 \odot}$, Tatyane Martins Cirilo ${ }^{10}$, Letícia \\ Pereira Bezerra $^{1}{ }^{\bullet}$, Laryssa Oliveria Silva ${ }^{1}$, Claudimary Bispo dos Santos ${ }^{50}$
}

${ }^{1}$ Laboratório de Parasitologia Humana e Malacologia, Universidade Estadual de Alagoas-UNEAL, Santana do ipanema-AL Brasil. ${ }^{2}$ Departamento de Parasitologia, Laboratório de Bioquímica de Tripanososmátideos, Instituto de Ciências Biomédicas, Universidade de São Paulo - USP, São Paulo-SP Brasil.

${ }^{3}$ Departamento de Morfologia, Centro de Ciências Biológicas e da Saúde, Universidade Federal de Sergipe, UFS, São Cristóvão-SE Brasil.

*Autor para correspondência, E-mail: israel.santos@uneal.edu.br

Resumo. Enteroparasitoses são um grupo de doenças provocadas por helmintos e protozoários, que vivendo na luz intestinal de crianças e adultos, determinam condições que podem culminar com déficit para o processo de escolarização. Assim, o objetivo deste estudo foi fazer um levantamento da prevalência de enteroparasitoses e fatores de risco para estas infecções em escolares de uma cidade do sertão alagoano. Para isso, uma amostra de fezes foi coletada e uma lâmina foi lida para cada participante. Um questionário semiestruturado foi aplicado para o conhecimento dos fatores de risco associados à infecção parasitária. dos 107 participantes do estudo, 48 destes (45\%) estavam parasitados por pelo menos uma espécie de parasita. As espécies de parasitas encontradas foram: Entamoeba coli $(39,58 \%)$, Entamoeba histolytica/dispar (29,16\%), Giardia lamblia (20,8\%), Endolimax nana (16,6\%), Iodamoeba butschilii (12,5\%), Trichuris trichiura (10,41\%), Hymenolepis nana (4,16\%), Ascaris lumbricoides (4,16\%) e Strongyloides stercoralis $(2,08 \%)$. Dos positivos, $52 \%$ eram do sexo feminino, $64,5 \%$ moravam em casa própria, $60,4 \%$ tinham o pai como chefe da família, $70,8 \%$ moravam em rua com esgoto correndo a céu aberto, $79,1 \%$ afirmaram lavar as mãos antes das refeições e $83,3 \%$ também após usar o banheiro, $75 \%$ possuíam o hábito de brincar em locais com terra e 64,5\% moravam em ruas com criação de animais, mas $72,9 \%$ possuíam o hábito de andar calçado. Nenhuma das variáveis analisadas apresentou associação estatística significativa com a variável dependente infecção parasitária. Assim, esse estudo evidencia que na cidade em estudo ainda há uma elevada prevalência da infecção discente por enteroparasitas.

Palavras chave: Helmintos, protozoários, parasitoses, saúde pública

\section{Enteroparasitosis and risk factors in schoolchildren from a city in northeastern Brazil}

Abstract. Enteroparasitosis are a group of diseases provocated by helmintes and protozoan, that living in the gut light of children and adults, determine in the first conditions can result with deficit for the process of schooling. Therefore, the objective this study was to do a survey of prevalence of enteroparasitosis and risk factors for these infections in schoolchildren of a city from alagoano hinterland. For that, a sample of feces was collected, and a blade was read for each participant. A semi-structured questionnaire was applicated 
for the knowledge of the risk factors associates the parasitological infection. Of the 107 participants of study, 48 these (45\%) were parasitized for at least one species of parasite. The species of parasites found were: Entamoeba coli (39,58\%), Entamoeba histolytica/dispar (29,16\%), Giardia lamblia (20,8\%), Endolimax nana (16,6\%), Iodamoeba butschilii (12,5\%), Trichuris trichiura (10,41\%), Hymenolepis nana (4,16\%), Ascaris lumbricoides (4,16\%) e Strongyloides stercoralis (2,08\%). Of the positives, 52\% were female gender, $64,5 \%$ lived in own home, $60,4 \%$ had father as chief of family, $70,8 \%$ lived in street with sewer running into the open sky, $79,1 \%$ claimed to handwash before meals and $83.3 \%$ also after to use the bathroom, $75 \%$ had the habit playing in places with earth and $64.5 \%$ lived in street with animal breeding, but $72.9 \%$ had the habit walk shoes. None of variables analyzed showed statistical association significative with the dependent variable parasitological infection. That, this study evidence that in the city studied still there is an elevated prevalence of student infection by enteroparasitosis.

Keywords: Helmintes protozoan, parasitosis, public health

\section{Enteroparasitosis y factores de riesgo en escolares de una ciudad del noreste de Brasil}

Resumen. Las enteroparasitosis son un grupo de enfermidades causadas por helmintos e protozoos, que viven en la luz intestinal de niños y adultos, determinan las primeras condiciones que puden culminar con um déficit en el processo de escolarización. Por lo tanto, el objetivo de este estudio fue hacer um levantamiento sobre la prevalência de enteroparasitosis y los factores de riesgo para estas infecciones em escolares de una ciudad en el interior de Alagoas. Para esto, se recogió una muestra de heces y se leyo uma lamina para cada participante. Se aplicó un cuestionario semiestructurado para conocer los factores de riesgo associados con la infección parasitaria. De los 107 participantes del estudio, 48 de estos (45\%) fueron parasitados por al menos una espécie de parásito. Las espécies de parasitos encontrados fueron: Entamoeba coli (39.58\%), Entamoeba histolytica/dispar (29.16\%), Giardia lamblia (20.8\%), Endolimax nana (16.6\%), Iodamoeba butschilii (12.5\%) Trichuris trichiura (10.41\%), Hymenolepis nana (4.16\%), Ascaris lumbricoides $(4.16 \%)$ y Strongyloides stercoralis (2.08\%). De los positivos, 52\% eran mujeres, 64.5\% vivían en su propria casa, $60.4 \%$ tenían a su padre como cabeza de familia, $70.8 \%$ vivían en la calle com alcantarillado al aire libre, $79.1 \%$ dijeron lavarse las manos antes de las comidas y también el $83.3 \%$ después de ir al baño, el $75 \%$ tenía la costumbre de jugar en lugares com tierra y el $64.5 \%$ vivía em las calles com cria de animales, pero el $72.9 \%$ tenía la costrumbre de andar con sapatos. Ninguna de las variables analizadas mostro una asociación estadísticamente significativa con la variable dependiente infección parasitaria. Por lo tanto, este estudio muestra que en la ciudad en estudio todavia existe un alta prevalência de infección estudiantil con enteroparásitos.

Palavras clave: Helmintos protozoos, parásitos, salud pública

\section{Introdução}

As enteroparasitoses constituem um subgrupo de parasitas que vivem na luz intestinal do hospedeiro humano (Costa-Macedo et al., 1998). Nesse ambiente, estes seres provocam desordens que culminam em quadro patológicos associados a um ou mais dos seguintes fatores: cepa do parasita, carga parasitária, além do estado imunológico e nutricional do hospedeiro. Além disso, essas parasitoses são uma grande causa da perda da qualidade de vida e desenvolvimento biológico de crianças nos países subdesenvolvidos e em desenvolvimento. Estima-se em 100.000 o número de mortes anuais causadas por protozoários e um bilhão de pessoas infectadas pelos helmintos Ascaris lumbricoides, Trichuris trichiura e Ancilostomídeos, este último sabidamente causador de anemia quando presente no aparelho digestivo do hospedeiro humano (Coura, 2015; Neves, 2005; Brasil 2005).

No Brasil, país em desenvolvimento, o índice de parasitoses é elevado (Pereira-Cardoso et al., 2010). E a relação entre infecção parasitária e desenvolvimento cognitivo e escolar continua sendo objeto de 
estudos de diversos grupos de pesquisa (Ezeamama et al., 2018; Santos et al., 2010), onde estas pesquisas demonstram a influência negativa da infecção parasitária sobre estes parâmetros de desenvolvimento biológico discente. Além disso, fazem-se necessários estudos mais aprofundados do fenômeno infecção parasitários na população em idade escolar para que se tenham dados referentes à prevalência de parasitas intestinais nesta população e os fatores de risco envolvidos na manutenção da prevalência, para que assim possam ser tomadas medidas profiláticas, pelo poder público de saúde, com vistas no melhor aproveitamento dos processos pedagógicos e no desenvolvimento cognitivo destes alunos.

O objetivo deste estudo foi fazer o levantamento da prevalência de enteroparasitoses e fatores de risco para estas infecções em escolares de uma cidade do sertão alagoano.

\section{Material e métodos}

\section{Delineamento do estudo}

Trata-se de um trabalho descritivo de corte transversal, realizado entre os meses de março a dezembro de 2014, com alunos do Ensino Fundamental II, de escolas públicas estaduais e municipais da zona urbana da cidade de Santana do Ipanema, Alagoas.

Esta cidade localiza-se a $207 \mathrm{~km}$ da capital do Estado, Maceió, e tem coordenadas geográficas $09^{\circ} 22^{\prime} 40^{\prime \prime}, 37^{\circ} 14^{\prime} 42^{\prime \prime}$, respectivamente latitude e longitude. Segundo o Instituto Brasileiro de Geografia e Estatística (IBGE, 2000), sua população estava estimada, para 2016, em 48.033 habitantes, possuindo um Índice de Desenvolvimento Humano (IDH) em 2010 de 0,591.

Inicialmente, a secretaria municipal de educação foi contatada para o levantamento do número de escolas na zona urbana da cidade. Em seguida, todas as escolas foram visitadas e os objetivos do estudo foram explicados aos dirigentes e estes assinaram um termo de participação da escola na pesquisa.

Foram registrados 414 alunos elegíveis para participação no estudo. Desse total, apenas 107 (25,8\%) constituíram a amostra efetiva estudada, pois os demais não entregaram o material fecal no dia agendado e no dia seguinte, dia de nova coleta, ou os responsáveis pelo aluno não permitiram a participação deste na pesquisa. Da mesma forma, das 15 escolas que ofertavam ensino fundamental II, apenas 9 tiveram alunos incluídos no estudo devido às questões mencionadas.

\section{Coleta e análise dos dados}

Os dados foram coletados por meio de dois procedimentos: entrega do material fecal (amostra única) e aplicação de um questionário estruturado com questões para conhecimento do perfil socioeconômico, hábitos culturais e de higiene dos participantes da pesquisa e de seus familiares.

Para a coleta do material fecal, inicialmente foi feita uma reunião/palestra com os pais ou responsáveis pelos alunos, em cada escola. Neste momento foram explicados os objetivos do estudo, a forma correta da coleta da amostral fecal e a assinatura do Termo de Consentimento Livre e Esclarecido (TCLE) por estes responsáveis. Posteriormente, em dias agendados, por escola, foram feitas a entrega e a busca do coletor previamente identificado com os dados dos participantes. Em caso de não comparecimento do aluno no dia da entrega do material fecal, ficou definido mais uma busca no dia seguinte. Todo o material entregue foi acondicionado em caixa de isopor contendo gelo e enviados ao Laboratório de Parasitologia Humana e Malacologia do Campus II - Santana do Ipanema, da Universidade Estadual de Alagoas.

Neste laboratório as amostras foram submetidas ao método de sedimentação espontânea (Hoffman et al., 1934). Foi confeccionada 1 (uma) lâmina para cada participante do estudo, e esta foi analisada em microscópio binocular, nas objetivas de $10 \mathrm{X}$ e $40 \mathrm{X}$. Os resultados foram expressos como "Ausência e/ou presença de ovos/cistos de determinado parasita na amostra analisada". Todos os resultados foram devolvidos aos responsáveis pelos alunos, que puderam encaminhar as suas crianças para um posto do PSF (Programa Saúde da Família) de sua comunidade para tratamento médico, no caso dos positivos.

$\mathrm{Na}$ análise de associação, a variável infecção parasitária foi assumida como depende; enquanto gênero, recebimento de auxílio do governo, tipo de moradia, chefe de família, esgoto correndo a céu 
aberto, tipo de cobertura das ruas (pavimentação ou outros), forma de armazenamento dos alimentos, hábito de lavar as mãos antes das refeições e após usar o banheiro, brincar em locais com terra e criação de animais nestes locais, hábito de andar calçado e forma de descarte do lixo doméstico foram consideradas variáveis independentes. Ainda em relação a esta análise, o número de pessoas por casa inicialmente coletada como variável quantitativa foi, em seguida, transformada em categórica.

Os dados foram computados e armazenados em planilha do Microsoft Excel, versão 2007. Houve dupla entrada dos dados, seguidas de conferência. Para os resultados, os dados são inicialmente apresentados de forma descritiva, por meio de médias e proporções. Em seguida, a análise estatística foi feita a partir do teste de associação qui-quadrado. Foi considerada associação das duas variáveis quando o valor do qui-quadrado calculado foi maior que o tabelado e a significância da associação quando a estatística do teste encontrou $\mathrm{P}<0,05$.

\section{Considerações éticas}

Este estudo foi enviado ao Comitê de Pesquisa com Seres Humanos da Universidade Estadual de Ciências da Saúde de Alagoas - UNCISAL, e foi aprovada e liberada de acordo com o Parecer $\mathrm{n}^{\circ}$ 611.835 de 19/03/2014.

\section{Resultados}

Na tabela 1 são apresentados os resultados do questionário e das análises parasitológicas.

Tabela 1. Relação entre a infecção parasitária e algumas variáveis sociais, econômicas e culturais de alunos de escolas públicas estaduais e municipais da cidade de Santana do Ipanema, Alagoas, observada no ano de 2014.

\begin{tabular}{|c|c|c|c|c|c|c|}
\hline \multirow{2}{*}{ Variáveis } & \multirow{2}{*}{ Categoria } & \multicolumn{4}{|c|}{ Parasitismo } & \multirow{2}{*}{ p-valor } \\
\hline & & Sim & $\%$ & Não & $\%$ & \\
\hline \multirow{2}{*}{ Gênero } & Masculino & 23 & 45,1 & 28 & 54,9 & 0,96 \\
\hline & Feminino & 25 & 44,6 & 31 & 55,4 & \\
\hline \multirow{2}{*}{ Moradia } & Própria & 31 & 44,9 & 38 & 55,1 & 0,98 \\
\hline & Alugada & 17 & 44,7 & 21 & 55,3 & \\
\hline \multirow{2}{*}{ Chefe da família } & Homem & 29 & 39,7 & 44 & 60,3 & 0,08 \\
\hline & Mulher & 19 & 57,6 & 14 & 42,4 & \\
\hline \multirow{2}{*}{ Auxílio do governo } & Sim & 44 & 44,0 & 56 & 56,0 & 0,49 \\
\hline & Não & 4 & 57,1 & 3 & 42,9 & \\
\hline \multirow{2}{*}{ Esgoto peridoméstico } & Sim & 34 & 44,7 & 42 & 55,3 & 0,96 \\
\hline & Não & 14 & 45,2 & 17 & 55,8 & \\
\hline \multirow{2}{*}{ Calçamento na rua } & Sim & 40 & 46,0 & 47 & 54,0 & 0,46 \\
\hline & Não & 7 & 36,8 & 12 & 63,2 & \\
\hline \multirow{2}{*}{ Armazenamento dos alimentos } & Embalagem original & 16 & 45,7 & 19 & 54,3 & 0,90 \\
\hline & Recipiente fechado & 32 & 44,4 & 40 & 55,6 & \\
\hline \multirow{2}{*}{$\mathrm{N}^{\mathrm{o}}$ de pessoas/casa } & Até 4 & 21 & 41,2 & 30 & 58,8 & 0,46 \\
\hline & 5 ou mais & 27 & 48,2 & 29 & 51,8 & \\
\hline \multirow{2}{*}{ Descarte do lixo doméstico } & Carro do lixo & 44 & 44,0 & 56 & 56,0 & 0,49 \\
\hline & Terreno baldio & 4 & 57,1 & 3 & 42,9 & \\
\hline \multirow{2}{*}{$\begin{array}{l}\text { Lavar as mãos após usar o } \\
\text { banheiro }\end{array}$} & Sim & 40 & 43,0 & 53 & 57,0 & 0,20 \\
\hline & Não & 8 & 61,5 & 5 & 38,5 & \\
\hline \multirow{2}{*}{$\begin{array}{l}\text { Lavar as mãos após antes das } \\
\text { refeições }\end{array}$} & Sim & 38 & 42,7 & 51 & 57,3 & 0,31 \\
\hline & Não & 10 & 55,6 & 8 & 44,4 & \\
\hline \multirow{2}{*}{$\begin{array}{l}\text { Brincar em locais com } \\
\text { terra/areia }\end{array}$} & Sim & 36 & 44,4 & 45 & 55,6 & 0,87 \\
\hline & Não & 12 & 46,2 & 14 & 53,8 & \\
\hline \multirow{2}{*}{ Andar calçado } & Sim & 35 & 45,4 & 42 & 54,6 & 0,84 \\
\hline & Não & 13 & 43,3 & 17 & 56,7 & \\
\hline \multirow{2}{*}{ Criação de animais no bairro } & Sim & 31 & 45,6 & 37 & 54,4 & 0,91 \\
\hline & Não & 16 & 44,4 & 20 & 55,6 & \\
\hline
\end{tabular}


Dos 107 alunos, $56(52,3 \%)$ eram do sexo feminino e 51 (47,7\%) do sexo masculino, as idades variaram entre 5 e 15 anos. Quarenta e oito alunos (45\%) estavam positivos para pelo menos uma espécie de parasita. Houve uma maior proporção de infecção por protozoário, 43 casos $(89,58 \%)$, do que por helminto, 5 casos $(10,42 \%)$. O monoparasitismo esteve presente em 26 indivíduos $(54,17 \%)$, enquanto o poliparasitismo em 22 indivíduos (45,83\%).

As espécies de parasitas encontradas no material fecal dos discentes foram: Entamoeba coli (39,58\%), Entamoeba histolytica/dispar (29,16\%), Giardia lamblia (20,8\%), Endolimax nana (16,6\%), Iodamoeba butschilii (12,5\%), Trichuris trichiura (10,41\%), Hymenolepis nana (4,16\%), Ascaris lumbricoides $(4,16 \%)$ e Strongyloides stercoralis $(2,08 \%)$.

Dos positivos, $52 \%$ eram do sexo feminino, $64,5 \%$ moravam em casa própria, $60,4 \%$ tinham o pai como chefe da família, 70,8\% moravam em rua com esgoto correndo a céu aberto, $79,1 \%$ afirmaram lavar as mãos antes das refeições e 83,3\% também após usar o banheiro, $75 \%$ possuíam o hábito de brincar em locais com terra e 64,5\% moravam em ruas com criação de animais, mas 72,9\% possuíam o hábito de andar calçado. Nenhuma variável apresentou associação estatisticamente significativa com a variável dependente infecção parasitária (Tabela 1).

\section{Discussão}

Esse trabalho demonstra a permanência de uma elevada prevalência de enteroparasitoses na amostra estudada. Em estudos anteriores, 2009 e 2010, realizados na cidade de Santana do Ipanema, essa prevalência foi de $86,6 \%$ e $64,0 \%$, respectivamente. Essa redução pode ter ocorrido por conta do tratamento em massa da população em idade escolar quando da implantação e implementação do Programa Saúde na Escola (PSE) a partir da adesão do município no ano de 2011 (Comunicação pessoal, dados não publicados). Além disso, considerando que entre 2009 e 2010 houve uma redução de 22,6\% na taxa de infecção dos alunos pesquisados, percebe-se uma redução muita pequena nessa taxa, no intervalo entre 2011 e 2014, pois neste último cenário a redução foi de apenas 19\%, o que significa que em média houve uma redução anual de $4,75 \%$ no último período analisado, muito menor do que os $22,6 \%$ do primeiro período.

Outro fato muito importante de se destacar foi a mudança de perfil do grupo parasitário diagnosticado nos alunos desta cidade. Em 2009 e 2010, havia uma prevalência considerável de helmintos no material analisado por ambas as equipes de pesquisadores. No entanto, como mencionado acima, a partir da implementação do PSE, que preconiza a administração em massa do albendazol, o número de diagnóstico por protozoário aumentou, o que demonstra que esta abordagem do Ministério da Saúde está surtindo efeito no que diz respeito à redução das espécies de helmintos encontradas parasitando indivíduos, sobretudo crianças, nas cidades brasileiras.

Vale ressaltar que essa mudança de perfil parasitário está sendo observado em outras localidades do país (Auler et al., 2018; Dias et al., 2018; Pereira et al., 2018). E este fato é de grande relevância, pois já há estudo propondo um déficit cognitivo e educacional para as crianças parasitadas por helmintos (Ezeamama et al., 2018). Contudo, resta ainda uma preocupação devido a essa mudança de perfil parasitário, pois já se sabe, também, que a infecção por protozoário pode ser igualmente deletéria para os processos de ensino e aprendizagem discente ( $\underline{\text { Santos et al., 2010)}}$.

Com relação aos possíveis fatores de risco abordados neste trabalho, não foi possível estabelecer uma associação destes com a variável infecção parasitária. Isto pode ter se dado devido a uma limitação do nosso estudo, tal seja: tamanho da amostra, número de amostra fecal coletada e/ou número de lâmina analisada por indivíduo. Outros estudos apontam muitos dos fatores aqui estudados como associados ao parasitismo (Ferreira et al., 2000; Zaiden et al., 2008), o que reforça ainda mais a nossa hipótese de limitação.

Em nosso estudo houve uma maior prevalência do protozoário comensal Entamoeba coli. Apesar do seu modo de vida não patogênico, a $E$. coli é um importante bioindicador de insalubridade e a sua presença sinaliza para o risco de contaminação por protozoários patogênicos, como $E$. histolytica e $G$. lamblia, ambos também detectados no material fecal de muitos dos alunos participantes deste trabalho, pois a forma de contaminação, fecal-oral, é igual para estes protozoários (Andreis et al., 2008; Macedo, 2005; Rocha et al., 2000; Saturnino et al., 2003). 
Dessa forma, e diante dos achados deste estudo, reforça-se a necessidade de educação em saúde na cidade pesquisada e em outras com características socioeconômicas semelhantes, pois apesar da acentuada redução do número de helmintos diagnosticados, isso provavelmente devido ao trabalho dos profissionais envolvidos no PSE, nota-se a presença persistente de parasitas protozoários, de forma de transmissão basicamente fecal-oral.

Assim, é recomendado que as equipes executoras deste programa possam implementar o que diz o Art. $4^{\circ}$, incisos XIV, XVI e XVII do Decreto $\mathrm{N}^{\circ}$ 6.286, de 5 de dezembro de 2007, onde há expressamente a recomendação do trabalho permanente em educação em saúde, pois essa medida aliada a medicalização, já estabelecida no âmbito deste programa, pode culminar com a promoção da saúde e a melhoria da qualidade de vida destes discentes (Ali et al., 2003).

Além disso, recomenda-se também o uso de outros métodos de diagnóstico para o conhecimento da real prevalência do parasitismo e das espécies de protozoários e helmintos de ocorrência nesta e em outras áreas de estudo, pois é sabido que Enterobius vermiculares não é facilmente diagnosticado pelo método aqui empregado (sedimentação espontânea), portanto podendo a sua prevalência ter sido subnotificada neste estudo, como também abordagens moleculares e/ou bioquímica para o conhecimento real da(s) espécie(s) de Entamoeba presente(s) na área, pois também já se sabe que pelo exame parasitológico de fezes não é possível fazer a diferenciação morfológica dos cistos de $E$. histolytica com os cistos de E. dispar, nem tampouco dos cistos de E. moshkovskii, estas duas últimas permanecendo controversas quanto à sua capacidade de patogenicidade para o ser humano (Ali et al., 2003).

\section{Conclusão}

Diante dos resultados apresentados neste estudo, pôde-se perceber que a prevalência da infecção parasitária ainda continua alta na cidade de Santana do Ipanema, Alagoas, tendo uma significativa redução de infecções helmínticas, quando comparados a estudos anteriores, o que contrasta com a alta prevalência de protozoários comensais e patogênicos encontrados. Além disso as variáveis sociais, econômicas e culturais estudadas aqui não apresentaram associação estatística significativa com o parasitismo na amostra estudada.

\section{Agradecimentos}

Os autores agradecem ao apoio da FAPEAL (Fundação de Amparo à Pesquisa de Alagoas), pela bolsa ofertada durante a realização desta pesquisa.

\section{Referências bibliográficas}

Ali, I. K. M., Hossain, M. B., Roy, S., Ayeh-Kumi, P. F., Petri Junior, W. A., Haque, R., \& Clark, C. G. (2003). Entamoeba moshkovskii infections in children in Bangladesh. Emerging Infectious Diseases, 9(5), 580-584.

Andreis, A., Schuh, G. M., \& Tavares, R. G. (2008). Contaminação do solo por parasitas e ocorrência de doenças intestinais. Revista EVS-Revista de Ciências Ambientais e Saúde, 35(6), 1169-1177.

Auler, M. E., Campos, L. A., Horst, J. A. E., dos Santos, T. B., Miyahara, C. T. S., Paula, C. R., Ruiz, L. S., Gandra, R. F., \& Figueiredo, D. L. A. (2018). Saúde itinerante nos centros municipais de educação infantil do município de Guarapuava-PR; os desafios da promoção da saúde em crianças expostas a doenças parasitárias. Arquivos de Ciências Da Saúde Da UNIPAR, 22(1), 33-41.

BRASIL. (2005) Ministério da Saúde. Secretaria de Vigilância em Saúde. Plano Nacional de Vigilância e Controle das enteroparasitoses, 42p.

BRASIL. (2007). Presidência da República. Casa Civil. Subchefia de Assuntos Jurídicos. Decreto $N^{o}$ 6.286, de dezembro de 2007. Brasília: Casa Civil.

Costa-Macedo, L. M., Machado-Silva, J. R., Rodrigues-Silva, R., Oliveira, L. M., \& Vianna, M. S. R. (1998). Enteroparasitoses em pré-escolares de comunidades favelizadas da cidade do Rio de Janeiro, Brasil. Cadernos de Saúde Pública, 14, 851-855.

Coura, J. R. (2015). Dinâmica das doenças infecciosas e parasitárias. In Dinâmica das doenças 
infecciosas e parasitárias (pp. 1175-2045). Guanabara Koogan.

Dias, M. G. P. F., Fregonesi, B. M., Zagui, G. S., Tonani, K. A. A., Julião, F. C., Beda, C. F., Machado, C. S., Machado, G. P., Silva, T. V., \& Segura-Muñoz, S. I. (2018). Enteroparasitos em crianças de instituição de ensino filantrópica: ênfase para Cryptosporidium spp. e Giardia spp. Arquivos de Ciências Da Saúde, 25(1), 51-55.

Ezeamama, A. E., Bustinduy, A. L., Nkwata, A. K., Martinez, L., Pabalan, N., Boivin, M. J., \& King, C. H. (2018). Cognitive deficits and educational loss in children with schistosome infection-a systematic review and meta-analysis. PLoS Neglected Tropical Diseases, 12(1), 1-23.

Ferreira, M. U., Ferreira, C. S., \& Monteiro, C. A. (2000). Tendência secular das parasitoses intestinais na infância na cidade de São Paulo (1984-1996). Revista de Saúde Pública, 34(6), 73-82.

Hoffman, W. A., Pons, J. A., \& Janer, J. L. (1934). The sedimentation-concentration method in schistosomiasis mansoni. 283-291.

IBGE. (2000). Instituto Brasileiro de Geografia e Estatística. Censo Demográfico: Santana do Ipanema. Brasil.

Macedo, H. S. (2005). Prevalência de parasitos e comensais intestinais em crianças de escolas da rede pública municipal de Paracatu (MG). Revista Brasileira de Análises Clínicas, 37(4), 209-213.

Neves, D. P. (2005). Parasitologia humana. Editora Atheneu.

Pereira-Cardoso, F. D., Araújo, B. M., Batista, H. L., \& Galvão, W. G. (2010). Prevalência de enteroparasitoses em escolares de 06 a 14 anos no município de Araguaína-Tocantins. Revista EEetrônica de Farmácia, 7(1), 54-64.

Pereira, G., Ribeiro, C., Costa, I., Silva, J., Calado, L., Nunes, B., Aguiar, J., Rodrigues, F., \& Mota, M. (2018). Prevalência de infecções parasitárias intestinais oriundas decrianças residentes em áreas periféricas, município de Juazeiro do norte-Ceará. Revista Interfaces: Saúde, Humanas e Tecnologia, 5(14), 21-27.

Rocha, R. S., Silva, J. G., Peixoto, S. V., Caldeira, R. L., Firmo, J. O. A., Carvalho, O. dos S., \& Katz, N. (2000). Avaliação da esquistossomose e de outras parasitoses intestinais, em escolares do município de Bambuí, Minas Gerais, Brasil. Revista Da Sociedade Brasileira de Medicina Tropical, 33(5), 431-436.

Santos, I. G. A., Cruz Júnior, A. R., Costa, L. M. L., \& Silva, W. C. M. (2010). Parasitoses como fator ambiental de interferência negativa nos processos de ensino e aprendizagem em duas escolas de Santana do Ipanema-AL. Revista Ambientale, 2(2), 58-64.

Saturnino, A. C. R. D., Nunes, J. F. L., \& Silva, E. M. A. (2003). Relaçäo entre ocorrência de parasitas intestinais e sintomatologia observada em crianças de uma comunidade carente de Cidade Nova, em Natal-Rio Grande do Norte, Brasil. Revista Brasileira de Análises Clínicas, 35(2), 85-87.

Zaiden, M. F., Santos, B. O., Cano, M. A. T., \& Nascif Júnior, I. A. N. (2008). Epidemiologia das parasitoses intestinais em crianças de creches de Rio Verde-GO. Medicina, 41(2), 182-187.

Recebido: 27 de dezembro, 2019.

Aprovado: 24 de fevereiro, 2020.

Publicado: 23 de abril, 2020.

Licenciamento: Este artigo é publicado na modalidade Acesso Aberto sob a licença Creative Commons Atribuição 4.0 (CC-BY 4.0), a qual permite uso irrestrito, distribuição, reprodução em qualquer meio, desde que o autor e a fonte sejam devidamente creditados. 